\title{
Drop formation due to turbulent primary breakup at the free surface of plane liquid wall jets
}

\author{
Z. Dai, W.-H.Chou, ${ }^{\text {a) }}$ and G. M. Faeth ${ }^{\text {b) }}$ \\ Department of Aerospace Engineering, The University of Michigan, Ann Arbor, Michigan 48109-2140
}

(Received 26 August 1997; accepted 23 January 1998)

\begin{abstract}
An experimental study of turbulent primary breakup at the free surface of plane liquid wall jets along smooth walls in still air at normal temperature and pressure is described. The study seeks a better understanding of spray formation processes in marine environments, such as in bow sheets. The measurements involved initially nonturbulent annular liquid wall jets, to approximate plane liquid wall jets, with the growth of a turbulent boundary layer along the wall initiated by a trip wire. Pulsed shadowgraphy and holography were used to observe liquid surface properties as well as drop sizes and velocities after turbulent primary breakup. Test conditions included several liquids (water, glycerol mixtures and ethyl alcohol), liquid/gas density ratios of 680-980, wall jet Reynolds numbers of $17000-840000$ and Weber numbers of 6100-57000, at conditions where direct effects of liquid viscosity were small. Measurements included the following: location of the onset of surface roughness, drop size and velocity distributions after breakup, flow properties at the onset of breakup, and mean drop sizes and velocities after breakup. In general, the measurements were correlated successfully based on phenomenological theories. (C) 1998 American Institute of Physics. [S1070-6631(98)01605-5]
\end{abstract}

\section{INTRODUCTION}

An experimental investigation of the liquid surface and drop formation properties of plane turbulent liquid wall jets along smooth walls in still air at normal temperature and pressure (NTP) is described. This flow is important as a model of spray formation processes in marine environments which affect the structure of both ship-generated and natural waves. The main objectives were to study the properties of liquid surfaces and sprays produced by turbulent liquid wall jets along smooth walls in still air, as follows: conditions for transformation to roughened liquid surfaces, drop size and velocity distribution functions after breakup, the properties (drop sizes and the location) of the onset of breakup, and the evolution of drop sizes after breakup as a function of distance along the liquid surface.

In spite of the complexities, there is general agreement about the qualitative features of spray formation for flow processes such as chutes, spillways, plunge pools, hydraulic jumps, bow sheets, open water waves and jets. ${ }^{1-3}$ The mechanism involves the propagation of vorticity (typically as turbulence) to the liquid surface, followed by the appearance of a turbulence-wrinkled interface between the liquid and the gas, and eventually by the formation of drops at the liquid surface (which will be called turbulent primary breakup in the following). The crucial role played by turbulence in such spray formation processes has been demonstrated by observations of turbulent and uniform nonturbulent liquid jets in still gases due to $\mathrm{Wu} e t a l{ }^{4}$ The turbulent liquid jets rapidly

\footnotetext{
a) Present address: Trane Co., LaCrosse, WI 54601.

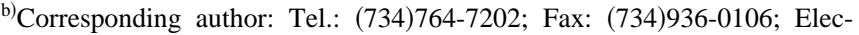
tronic mail: gmfaeth@umich.edu
}

developed wrinkled surfaces that subsequently broke up to form sprays. The properties of these sprays were strong functions of the turbulence properties of the liquid as expected for turbulent primary breakup processes but were relatively independent of the density of the surrounding gas for liquid/ gas density ratios, $\rho_{f} / \rho_{g}>500$; this behavior suggests small effects of aerodynamic forces at the liquid surface on spray properties. In contrast, the uniform nonturbulent jets behaved similar to liquid cutting jets and maintained smooth surfaces, with no evidence of breakup into a spray, within the region of observations.

The results of Wu et al. ${ }^{4}$ strongly suggest that the turbulence causing liquid surface roughness and turbulent primary breakup in wall jets originates from liquid motion along the wall. The process is sketched in Fig. 1 as it might be encountered for a ship-generated wall jet or bow sheet. The liquid flow along the wall then involves a relatively inconsequential laminar boundary layer (for typical practical applications), followed by transition to a growing turbulent boundary layer. The onset of liquid surface roughness is thought to correspond to conditions where the outer edge of the turbulent boundary layer approaches the liquid surface, however, this condition has not been quantified in terms of boundary layer properties during past studies. ${ }^{1-3}$ Subsequently, turbulent primary breakup begins and evolves with increasing distance along the surface of the turbulent wall jet. Earlier work considering such turbulent primary breakup processes will be considered next.

Turbulent primary breakup has been recognized as a mechanism of spray atomization since the early studies of De Juhasz et al. ${ }^{5}$ and Lee and Spencer. ${ }^{6,7}$ In particular, these studies showed that liquid turbulence properties at the jet exit affected the atomization and mixing properties of round liq- 


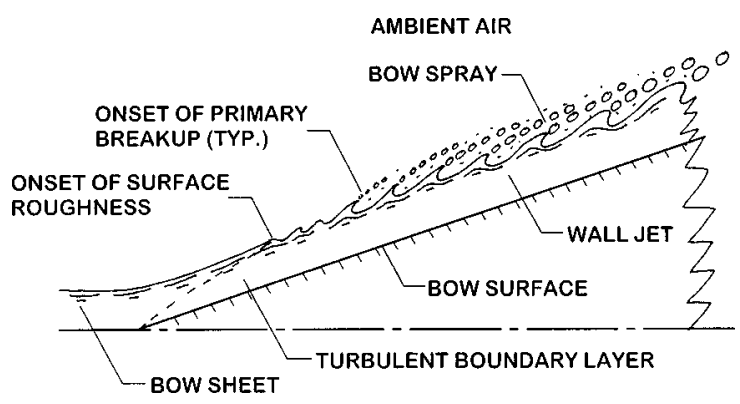

FIG. 1. Sketch of turbulent primary breakup process in a plane wall jet.

uid jets in still gases at NTP. Subsequently, Grant and Middleman, ${ }^{8}$ Phinney, ${ }^{9}$ and McCarthy and Malloy, ${ }^{10}$ observed that liquid jet stability and the onset of jet breakup were affected by turbulence at the jet exit. Finally, Hoyt and Taylor ${ }^{11,12}$ carried out a series of experiments with both coflowing and counterflowing round gas/liquid jets at NTP, that provided strong evidence that aerodynamic forces at the liquid surface did not have a large effect on turbulent primary breakup, as discussed earlier.

More details about the properties of turbulent primary breakup have been obtained during the recent studies of Ruff et al. ${ }^{13}$ Tseng et al. ${ }^{14}$ and $\mathrm{Wu}$ and co-workers. ${ }^{15-17}$ These studies involved pulsed shadowgraphy and holography to observe turbulent primary breakup of round free liquid jets in still gases with fully developed turbulent pipe flow at the jet exit. The results showed that the drop properties after turbulent primary breakup were related to the properties of the liquid-phase turbulence and yielded correlations for the onset and end of drop formation along the liquid surface as well as the evolution of drop size and velocity distributions with distance along the surface. Observations of liquid jets in various gas environments also established that aerodynamic effects at the liquid surface did not affect turbulent primary breakup properties for large liquid/gas density ratios, as noted earlier.

The objective of the present investigation was to extend the studies of turbulent primary breakup for round free liquid jets in still gases, ${ }^{13-17}$ to consider the same properties for plane liquid wall jets along smooth surfaces in still gases, using similar methods. The main issue was to resolve the effect of the fundamentally different turbulence properties of these two flows (a developing plane turbulent wall jet as opposed to a decaying round turbulent free jet) on the appearance of a roughened liquid surface and the outcome of turbulent primary breakup. A preliminary report of the study, emphasizing flow visualization and the onset of a roughened liquid surface and of turbulent primary breakup, using small aspect ratio annular liquid wall jets to approximate the properties of plane liquid wall jets, is presented by Dai et al. ${ }^{19}$ The present paper provides more extensive results for a broader range of aspect ratios (better approximating plane liquid wall jets) and flow properties as well as information about drop size and velocity distributions and the evolution of mean drop sizes and velocities along the liquid surface. Similar to earlier work, ${ }^{13-18}$ phenomenological analyses

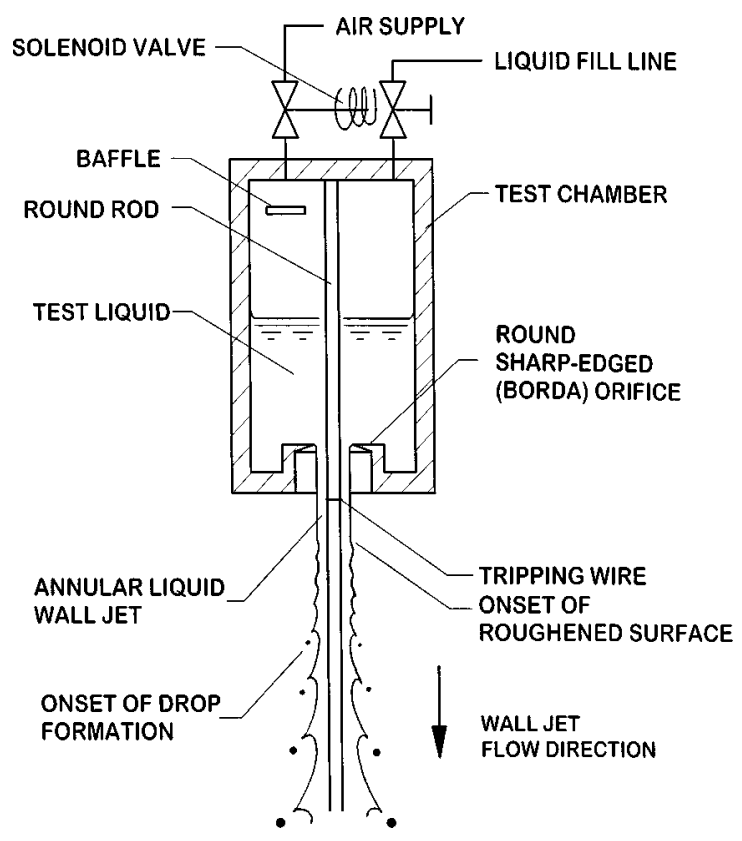

FIG. 2. Sketch of test apparatus for measurements of turbulent primary breakup in plane wall jets.

were used to help interpret and correlate the new measurements.

The paper begins with consideration of experimental methods. Results are then described, treating flow visualization, surface velocities, onset of surface roughness, drop size and velocity distributions after turbulent primary breakup, mean and fluctuating drop velocities after turbulent primary breakup, the onset of turbulent primary breakup and the variation of drop sizes after turbulent primary breakup with distance along the surface, in turn.

\section{EXPERIMENTAL METHODS}

\section{A. Apparatus}

A sketch of the apparatus used to measure the properties of turbulent primary breakup in plane wall jets appears in Fig. 2. All components of the apparatus in contact with the test liquids were fabricated from type 304 stainless steel. The test liquid was placed within a cylindrical test chamber that had a round sharp-edged (Borda) orifice at its bottom. Combined with a round rod passing down the axis of the test chamber, this arrangement provided an initially nonturbulent annular wall jet along the rod, in much the same manner that $\mathrm{Wu}$ et al. ${ }^{4}$ used Borda orifices to generate nonturbulent round liquid jets. This chamber was partially filled with liquid while using an annular cork in the Borda orifice passage to prevent premature liquid outflow. The liquid was then forced through the orifice, ejecting the cork down the rod at the start of flow, by admitting high-pressure air to the top of the test chamber through a solenoid-actuated valve; a baffle near the air inlet prevented undesirable aeration of the liquid. The solenoid valve was closed at the end of liquid delivery so that the test chamber could be vented to the ambient pressure. The annular cork was then replaced so that the system could be refilled with liquid for the next test. 
The high pressure air was obtained from laboratory supplies and was stored in a $0.12 \mathrm{~m}^{3}$ air accumulator at pressures up to 1.4 MPa prior to a test. Two different test chambers were used having inside diameters of 50 and $165 \mathrm{~mm}$ and lengths of 150 and $305 \mathrm{~mm}$, respectively. The small and large chambers used rod diameters, $D_{\text {rod }}$, of 6.4 and 50.8 $\mathrm{mm}$, respectively, in order to evaluate effects of wall jet aspect ratio. Borda orifices of various diameters were used with these rods to provide orifice annulus widths, $b$, of 2.3, 3.8, 4.3 , and $7.0 \mathrm{~mm}$. Injection was vertically downward with the liquid captured in a baffled tub in order to prevent drops from splashing back up into the test area and obscuring the measurements. The entire test chamber and rod assembly could be moved vertically, with a accuracy of $0.5 \mathrm{~mm}$, in order to accommodate rigidly-mounted optical instrumentation.

The growth of a turbulent boundary layer along the wall (the rod) was initiated using a trip wire so that this position could be located accurately. The trip wires had diameters, $k$, in the range $0.1-0.5 \mathrm{~mm}$ so that $k / b<10 \%$. The laminar boundary layer thickness at the location of the trip wire was also estimated to be less than $10 \%$ of the annulus thickness. By maintaining these two conditions, separation of the wall jet from the rod due to the presence of the trip wire was avoided. It was also necessary to provide large enough values of $u^{*} k / \nu_{f}$, where $u^{*}$ is the friction velocity and $\nu_{f}$ is the kinematic viscosity of the liquid, to insure that transition to a turbulent boundary layer actually occurred at the trip wire; this issue will be discussed in more detail later.

Flow development times for the present wall jets were relatively short, 6-70 ms; therefore, relatively short injection times of 100-400 ms were adequate. In addition, present optical methods required times less than $0.1 \mathrm{~ms}$ for triggering and data acquisition and did not impose any limitations on flow times. Jet velocities were calibrated in terms of the pressure drop across the Borda orifice by measuring liquid surface velocities from double-pulse shadowgraphs.

\section{B. Instrumentation}

Instrumentation consisted of single and double-pulse shadowgraphy and holography. Arrangements and methods will be described only briefly because they are similar to past work, see Refs. 15-17. The light sources for both systems were frequency doubled YAG lasers (Spectra Physics Model GCR-130, $532 \mathrm{~nm}$ wavelength, $7 \mathrm{~ns}$ pulse duration, and up to $300 \mathrm{~mJ}$ per pulse) that could be controlled to provide pulse separations as small as $100 \mathrm{~ns}$ (pulse separations were measured with a digital oscilloscope). Pulsed shadowgraphy was used to measure flow properties extending up to a point just beyond the onset of breakup where drop concentrations were relatively small. The laser beams were expanded to provide a $40 \mathrm{~mm}$ diameter collimated beam that passed through the region being observed. The shadowgraphs were recorded using a $100 \times 125 \mathrm{~mm}$ film format at magnifications of $2-7$, with the camera focused at the median plane of the annular wall jet. These photographs were obtained with an open camera shutter under darkroom conditions so that the flash duration controlled the exposure time. Laser pulse times were short enough to stop liquid surface and drop motion. The use of different pulse strengths allowed directional ambiguity to be resolved due to the different contrast of the initial and final pulses. Data was obtained from the photographs by mounting them on a computer controlled $x-y$ traversing system (having a $1 \mu \mathrm{m}$ resolution) and observing the images with an MTI Model 65 video camera.

An off-axis holographic arrangement was used with optical penetration properties improved by reducing the diameter of the object beam through the flow by $3: 1$ and subsequently expanding it back to the size of the $100 \mathrm{~mm}$ diameter reference beam when the two beams were optically mixed to create the hologram. The present arrangement allowed drops as small as $5 \mu \mathrm{m}$ diameter to be observed and as small as 10 $\mu \mathrm{m}$ to be measured with $10 \%$ accuracy. The holograms were reconstructed using a $35 \mathrm{~mW}$ HeNe laser with the laser beam collimated at a $50 \mathrm{~mm}$ diameter and passed through the hologram to provide a real image of the spray. Analysis of the hologram images was the same as the shadowgraphs except that $x-y$ traversing of the hologram was supplemented by $z$ traversing (with $5 \mu \mathrm{m}$ resolution) of the video camera to allow for the three-dimensional nature of hologram reconstruction.

Methods of data reduction involved human-aided measurements of drop properties were the same as $\mathrm{Wu}$ and co-workers. ${ }^{15-17}$ Experimental uncertainties were evaluated using standard statistical methods, see Refs. 15-17 for the details. Irregular drops were assumed to be ellipsoids and were assigned diameters, $d$, equal to the diameter of a sphere having the same volume as the ellipsoid. Experimental uncertainties due to this definition of drop diameters are difficult to quantify; otherwise, experimental uncertainties $(95 \%$ confidence) of individual drop diameter measurements are less than $10 \%$ for drop diameters larger than $10 \mu \mathrm{m}$ (which comprised most of the present measurements). Measurements of mass median, MMD, and Sauter mean, SMD, diameters were obtained by summing over $40-400$ objects at each condition to obtain experimental uncertainties $(95 \%$ confidence) less than $15 \%$, mainly dominated by sampling limitations (note that the SMD is the diameter of a drop having the same surface-area/volume ratio as the spray as a whole).

Measurements of drop velocities were based on the motion of the centroid of the drops for the measured time period of the double pulses while summing over 40-250 objects to find mass-averaged mean and fluctuating streamwise, $\widetilde{u}, \vec{u}$ and cross-stream, $\widetilde{v}, \vec{v}^{\prime \prime}$ velocities. Experimental uncertainties $(95 \%$ confidence) of mass-averaged velocities were dominated by sampling limitations and are estimated as follows: streamwise mean velocities less than $10 \%$, streamwise and cross-stream velocity fluctuations less than $20 \%$ and cross-stream mean velocities less than $20 \%$. Finally, measurements of the locations of the onset of surface roughness and the onset of turbulent primary breakup were obtained as the average of 10 experiments; the experimental uncertainties (95\% confidence) of these determinations were relatively large, less than $30 \%$, due to sampling limitations. 
TABLE I. Summary of test conditions. ${ }^{\mathrm{a}}$

\begin{tabular}{ll}
\hline \multicolumn{1}{c}{ Parameter } & \multicolumn{1}{c}{ Range } \\
\hline$b(\mathrm{~mm})$ & $2.3,3.8,4.3$ and 7.0 \\
$D_{\text {rod }}(\mathrm{mm})$ & 6.4 and 50.8 \\
Liquids & water, glycerols, ethyl alcohol \\
$\rho_{f} / \rho_{g}$ & $680-980$ \\
$\mu_{f}(\mathrm{~kg} / \mathrm{m} \mathrm{s})$ & $0.0009-0.010$ \\
$\sigma(\mathrm{N} / \mathrm{m})$ & $0.022-0.071$ \\
$b_{f r} / b$ & $0.6-0.7$ \\
$\bar{u}_{s r}(\mathrm{~m} / \mathrm{s})$ & $15-47$ \\
$\operatorname{Re}_{f D}\left(\rho_{f} \bar{u}_{s r} D / \mu_{f}\right)$ & $17000-840000$ \\
$\mathrm{We}_{f D}\left(\rho_{f} \bar{u}_{s r}^{2} D / \sigma\right)$ & $6100-57000$ \\
$\mathrm{Oh}_{f D}\left(\mu_{f} /\left(\rho_{f} D \sigma\right)^{1 / 2}\right)$ & $0.001-0.013$ \\
\hline \hline
\end{tabular}

${ }^{\mathrm{a}}$ Liquid wall jet in air at $98.7 \mathrm{kPa}$ and $294 \pm 2 \mathrm{~K}$. Properties of air: $\rho_{g}$ $=1.18 \mathrm{~kg} / \mathrm{m}^{3}$ and $\mu_{g}=18.5 \times 10^{-6} \mathrm{~kg} / \mathrm{m} \mathrm{s}$.

${ }^{\mathrm{b}}$ Glycerin concentrations of $21 \%, 42 \%$ and $63 \%$ by mass.

\section{Test conditions}

Test conditions are summarized in Table I. Test liquids included water, glycerol mixtures $(21 \%, 42 \%$, and $63 \%$ glycerin by mass) and ethyl alcohol with air at NTP as the ambient gas. This provided values of $\rho_{f} / \rho_{g}$ of $680-980$, liquid viscosities, $\mu_{f}$, in the range $0.0009-0.010 \mathrm{~kg} / \mathrm{m} \mathrm{s}$ and values of the surface tension, $\sigma$, in the range $0.022-0.071 \mathrm{~N} / \mathrm{m}$. Liquid properties just prior to the onset of a roughened liquid surface included normalized wall jet heights, $b_{f r} / b$, of $0.6-$ 0.7 and average liquid surface velocities, $\bar{u}_{s r}$ of $15-47 \mathrm{~m} / \mathrm{s}$. For purposes of normalization, the hydraulic diameter, $D$, of the wall jets were based on the wall jet height, as follows:

$$
D / b_{f r}=4\left(1+b_{f r} / D_{\text {rod }}\right) .
$$

Characteristic Reynolds, $\mathrm{Re}_{f D}$, Weber, $\mathrm{We}_{f D}$ and Ohnesorge, $\mathrm{Oh}_{f D}$, numbers based on liquid properties and the hydraulic diameter, are defined in Table I. Values of $\mathrm{Re}_{f D}$ are in the range $17000-840000$, which provides the reasonably turbulent wall jet conditions needed for turbulent primary breakup, ${ }^{4}$ with the larger values approaching conditions representative of practical bow sheets. On the other hand, values of $\mathrm{Oh}_{f D}$ are relatively small, in the range 0.001-0.013, which implies relatively small direct effects of liquid viscosity on turbulent primary breakup; such behavior is also representative of most bow sheets encountered in practice.

\section{RESULTS AND DISCUSSION}

\section{A. Flow visualization}

The qualitative behavior of the onset of a roughened liquid surface, the subsequent development of liquid surface distortions, the properties of the onset of turbulent primary breakup, and the subsequent development of the breakup process along the liquid surface, can be seen in the pulsed shadowgraph photograph of Fig. 3. This test condition involves a $63 \%$ glycerol mixture with an annulus width of 2.3 $\mathrm{mm}$, a rod diameter of $6.4 \mathrm{~mm}$ and $\bar{u}_{s r}=17.3 \mathrm{~m} / \mathrm{s}$, which implies $\mathrm{Re}_{f D}=179000, \mathrm{We}_{f D}=50000$, and $\mathrm{Oh}_{f D}=0.013$. The trip wire was located slightly above the top of the left-

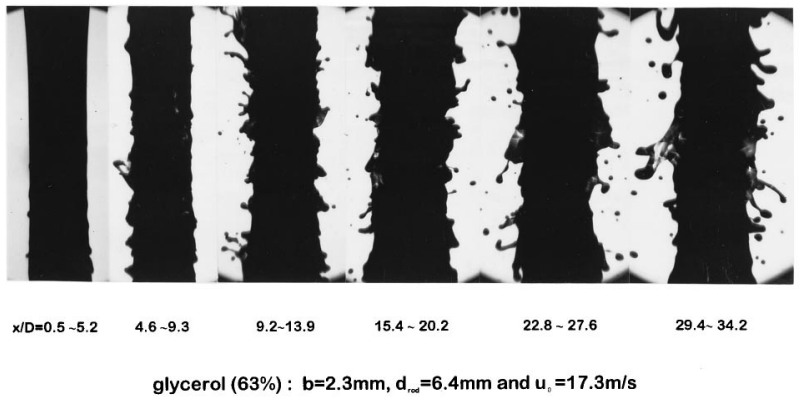

FIG. 3. Pulsed shadowgraphs of the turbulent primary breakup process in a plane wall jet: glycerol $(63 \%), b=2.3 \mathrm{~mm}, D_{\text {rod }}=6.4 \mathrm{~mm}$ and $\bar{u}_{s r}$ $=17.3 \mathrm{~m} / \mathrm{s}$. These conditions imply $\operatorname{Re}_{f D}=179000$, $\mathrm{We}_{f d}=50000$ and $\mathrm{OH}_{f D}=0.013$.

most shadowgraph, with progressively increasing distance, $x$, from the trip wire proceeding from top-to-bottom and leftto-right as noted below the images.

The capability of the sharp-edged Borda orifice to generate a turbulence-free initial flow is evident from the smooth liquid surface of the flow for some distance downstream of the trip wire seen in Fig. 3. For these conditions, the boundary layer along the wall is laminar prior to reaching the trip wire, with growth of a turbulent boundary layer beginning along the wall at the location of the trip wire.

The liquid surface in Fig. 3 remains smooth for a time as distance increases past the location of the trip wire. The onset of surface roughness is finally observed, however, toward the bottom of the left-most shadowgraph. Once liquid surface roughness appears, both the degree of surface roughness and the size of surface roughness elements increase with increasing streamwise distance from the trip wire. The roughness elements eventually become surprisingly long, as ligaments protruding from the liquid surface. These ligaments eventually break up at their tips, however, to form drops at the onset of turbulent primary breakup. Subsequent increases of distance into the turbulent primary breakup region yields progressively increasing ligament diameters, ligament lengths, and drop sizes after turbulent primary breakup. These trends are very similar to past observations of turbulent primary breakup for round liquid jets in still gases at conditions where effects of aerodynamic forces at the liquid surface are small. ${ }^{15-17}$

\section{B. Surface velocities}

The present wall jets are in a uniform pressure field adjacent to a still gas. As a result, wall jet velocities tend to decay due to flow resistance at both the wall and the gas/ liquid interface. Thus, measurements of the mean surface velocities of the wall jets were undertaken as a function of distance along the surface, in order to help define wall jet flow properties.

Typical measurements of wall jet surface velocities are plotted in Fig. 4. These test conditions consisted of water wall jets with $D_{\text {rod }}=50.8 \mathrm{~mm}$ and $b=3.8 \mathrm{~mm}$. Values of the mean surface velocity, $\bar{u}_{s}$, normalized by the mean surface velocity at the onset of surface roughness, $\bar{u}_{s r}$, are plotted as a function of streamwise distance from the mean location of 


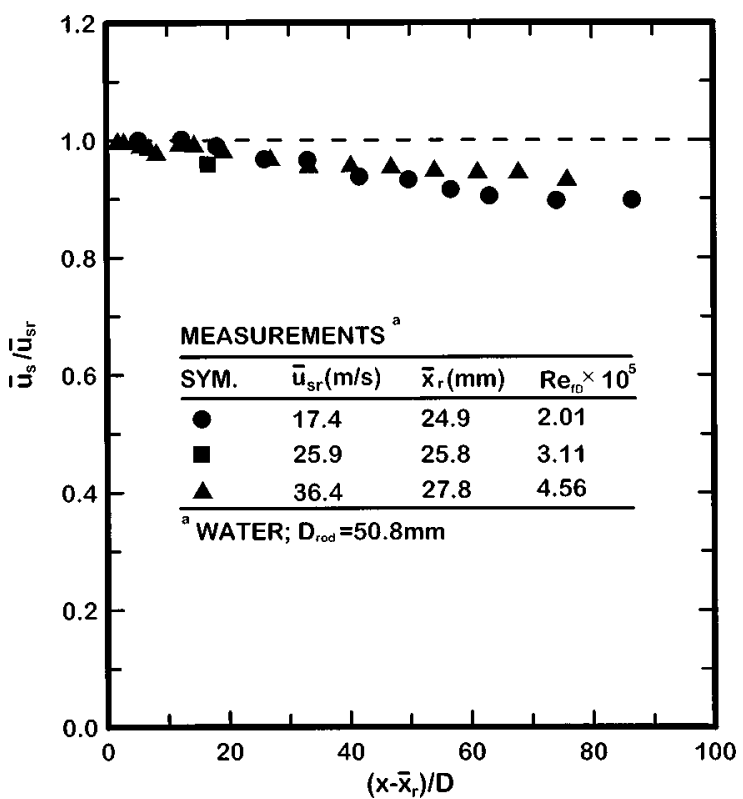

FIG. 4. Variation of liquid surface velocity with distance from the onset of surface roughness for plane wall jets.

the onset of surface roughness, normalized by the hydraulic diameter, $\left(x-\bar{x}_{r}\right) / D$. Measurements are shown for three strongly turbulent wall jets having characteristic Reynolds numbers in the range $200000-460000$. The variation of $\bar{u}_{s} / \bar{u}_{s r}$ with normalized distance is similar for all three flows, yielding roughly a $10 \%$ reduction of the surface velocity over a streamwise distance of 100 hydraulic diameters. Based on the definition of $D$ provided by Eq. (1), this implies only a $10 \%$ reduction of mean surface velocities for streamwise distances of up to roughly 400 wall jet heights. Thus, decay of velocities is not significant for the present wall jets and $\bar{u}_{s r}$ will be used to represent wall jet velocities. One exception to this practice will be the use of local mean surface velocities to normalize drop velocities after turbulent primary breakup, in order to assess relationships between drop and liquid surface velocities more precisely.

\section{Onset of surface roughness}

As discussed in connection with Fig. 2, the onset of surface roughness is thought to occur when the thickness of growing boundary layer along the wall becomes comparable to the thickness of the wall jet. This hypothesis was explored for three cases by Dai and Faeth: ${ }^{18}$ (1) an untripped laminar boundary layer reaches the surface first, (2) an untripped laminar boundary layer makes the transition to a turbulent boundary layer which reaches the surface first, and (3) a tripped turbulent boundary layer reaches the surface first. Only the last condition proved to be relevant for the present tripped boundary layers at relatively large Reynolds number conditions; therefore, the following discussion will be limited to this case. For these considerations, boundary layer development within the wall jet will be simplified considerably, as follows: Effects of the free surface on boundary layer growth will be ignored, variations of wall jet velocities and thickness will be ignored, justified by the results dis-

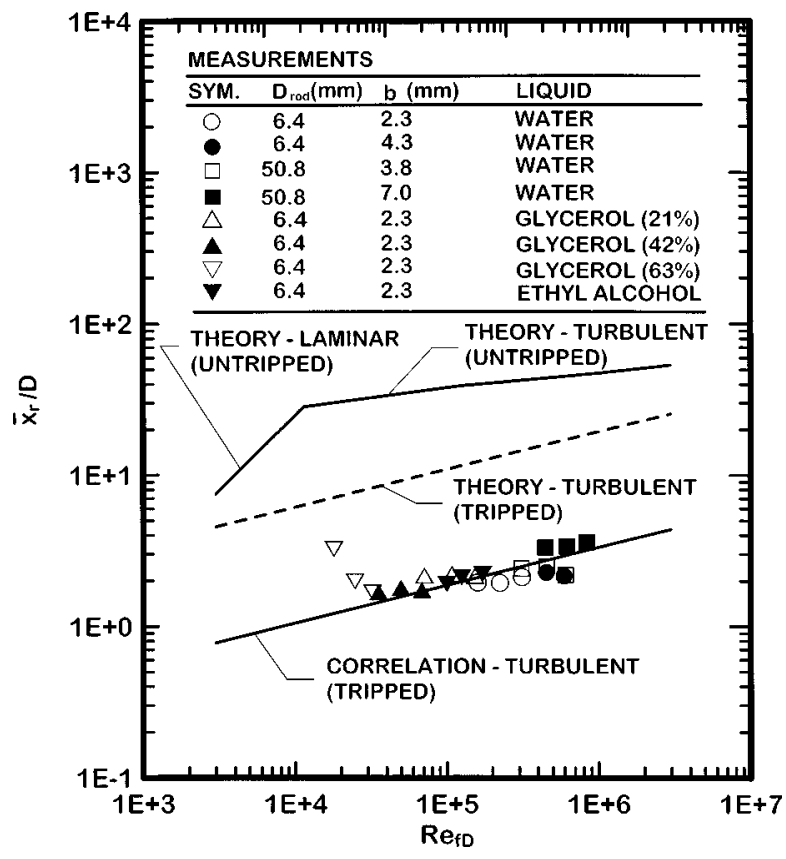

FIG. 5. Influence of Reynolds number on the onset of surface roughness for plane wall jets.

cussed in connection with Fig. 4; aerodynamic effects will be ignored, as discussed earlier; the wall surface will be assumed to be smooth, which is a condition of the present experiments; liquid properties will be assumed to be constant, which is appropriate for the present experiments; and turbulent boundary layer growth is initiated by the trip wire, where it is assumed that the boundary layer thickness, $\delta$ $=0$ at $x=0$. In the following, $\delta$ will be taken to be the cross-stream position where the streamwise mean velocity reaches $99 \%$ of $\bar{u}_{s r}$. Then, the condition where the outer edge of the turbulent boundary layer begins to interact with the wall jet is given by $b_{f r}=C_{r} \delta$ at $x=\bar{x}_{r}$, where $C_{r}$ is an empirical constant to account for the fact that $\delta$ is only a measure of the boundary layer thickness, etc. Finally, it is assumed that growth of the turbulent boundary layer can be based on Schlichting, ${ }^{19}$ assuming a $1 / 7$ th velocity distribution power law, which is reasonable for the present range of Reynolds numbers. Given these results, the expression for the streamwise distance where the turbulent boundary layer begins to interact with the liquid surface becomes

$$
\bar{x}_{r} / D=3.46\left(C_{r} b_{f r} / D\right)^{5 / 4} \operatorname{Re}_{f D}^{1 / 4},
$$

where $b_{f r} / D$ is given by Eq. (1).

Present measurements of $\bar{x}_{r}$ are plotted as suggested by Eq. (2) in Fig. 5, considering results for various test liquids over the available range of test conditions. Several predictions (correlations) are also shown on the plot, as follows: predictions for an untripped boundary layer involving transition from laminar to turbulent flow with $C_{r}=1$ and $D / b_{f r}$ $=4$ from Dai and Faeth, ${ }^{18}$ predictions for a turbulent boundary layer from Eq. (2) with $C_{r}=1$ and $D / b_{f r}=4$, and a bestfit correlation for a tripped turbulent boundary layer from Eq. (2) based on the present measurements. The two theoretical predictions are shown for illustrative purposes only; in par- 
ticular, the laminar/turbulent correlation is not representative of present test conditions where a trip wire is used to create an initially turbulent boundary layer at $x=0$. The prediction for a tripped turbulent boundary layer with $C_{r}=1$ also significantly overestimates $\bar{x}_{r} / D$, which suggests that disturbed surfaces appear when the turbulent boundary layer thickness is not a large fraction of the wall jet thickness. This behavior will be quantified next.

The correlation of present measurements appearing in Fig. 5, based on Eq. (2), is as follows:

$$
\bar{x}_{r} / D=0.061 \operatorname{Re}_{f D}^{1 / 4}, \quad \operatorname{Re}_{f D}>30000,
$$

where the criterion $\operatorname{Re}_{f D}>30000$ for the onset of effects associated with fully turbulent wall jets is very similar to the analogous criterion of $\mathrm{Wu}$ et $a{ }^{4}{ }^{4}$ for fully turbulent free jets. When $\operatorname{Re}_{f D}<30000, \bar{x}_{r} / D$ increases from the estimates of Eq. (3), due to effects of poorly developed turbulence in the boundary layer along the wall. The correlation of Eq. (3) corresponds to $C_{r}=0.2$ in Eq. (2) which is on the order of unity as anticipated for an empirical parameter of this type. This result also suggests that the present surface disturbances appear at distances well beyond the region typically assumed to involve turbulent wall boundary layer flows, e.g., at $\delta / b_{f r}=0.2$. It is also very helpful to note that the correlation of Eq. (1), based on the hydraulic diameter, is effective for aspect ratios in the range $D_{\text {rod }} / b=1.5-13.4$.

In addition to $\operatorname{Re}_{f D}$, the height of the trip wire influenced the onset of a roughened liquid surface. This effect was correlated following Ref. 18, based on the wire diameter and the friction velocity, $u^{*}$, where ${ }^{19}$

$$
u^{*}=\left(\tau_{w k} / \rho_{f}\right)^{1 / 2}
$$

and the wall shear stress at the trip wire location, $\tau_{w k}$, is given by

$$
\tau_{w k} /\left(\rho_{f} \bar{u}_{s r}^{2}\right)=0.332 / \operatorname{Re}_{f k},
$$

where $\operatorname{Re}_{f k}=\bar{u}_{s r} \bar{x}_{k} / \nu_{f}$ is the characteristic Reynolds number based on liquid properties and the streamwise distance, $\bar{x}_{k}$, between the Borda orifice and the trip wire.

Present measurements of the effect of trip wire Reynolds numbers on $\bar{x}_{r}$ are plotted in Fig. 6. The coordinates of this plot have been selected based on Eq. (3), where $\bar{x}_{r} /\left(D \mathrm{Re}_{f D}^{1 / 4}\right)$ should be a constant based on present measurements of the onset of surface roughness for a tripped turbulent boundary layer developing along the wall; this correlation is also illustrated on the figure. Aside from one outlier for a water wall jet at the lowest trip wire Reynolds number considered for this liquid, the results of Fig. 6 suggest little effect of trip wire properties for $u^{*} k / \nu_{f}>50$. At smaller values of $u^{*} k / \nu_{f}$, however, $\bar{x}_{r}$ progressively increases as the trip wire Reynolds number decreases because the wire disturbance becomes too weak to initiate a fully turbulent wall boundary layer.

\section{Drop size distributions}

Typical measurements of drop size distributions after turbulent primary breakup are illustrated in Fig. 7. These results are for wall jets at a number of different Reynolds

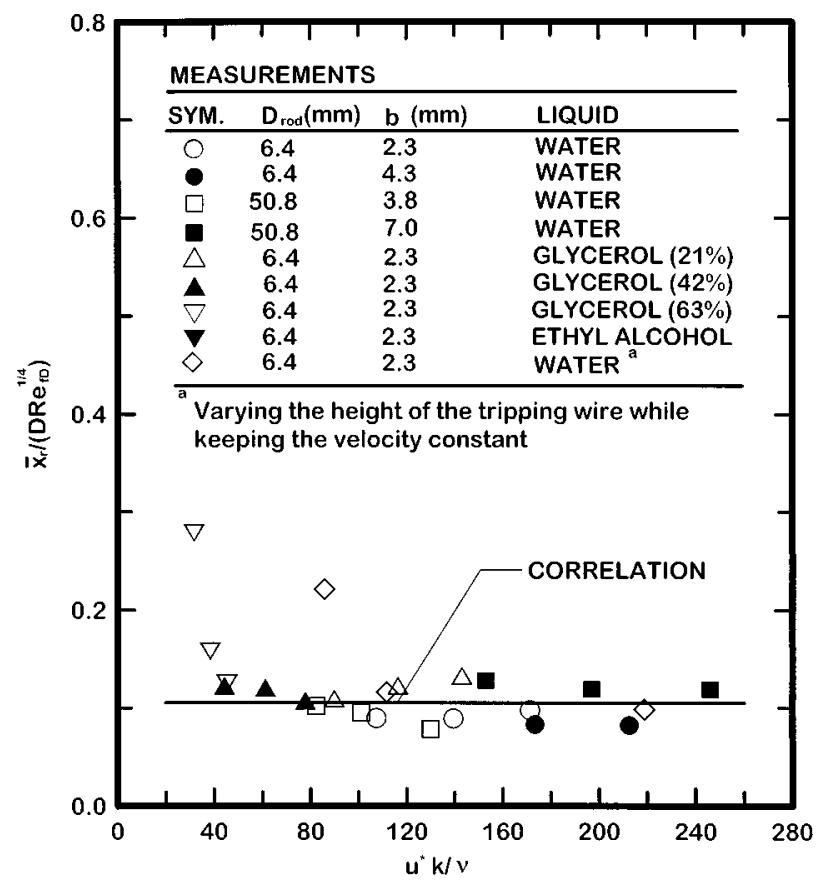

FIG. 6. Influence of trip wire properties on the onset of surface roughness for plane wall jets.

numbers and distances after the onset of turbulent primary breakup, but they are representative of behavior over the present test range. The results are plotted to test whether they satisfy the universal root normal distribution function with $\mathrm{MMD} / \mathrm{SMD}=1.2$ which Simmons $^{20}$ has shown is reasonably effective for correlating the drop size distributions of practical sprays. The present results clearly do satisfy the universal root normal distribution function, similar to a variety of primary $^{13-17}$ and secondary ${ }^{21,22}$ breakup processes. The root normal distribution function has only two moments; therefore, taking the best estimate of $\mathrm{MMD} / \mathrm{SMD}=1.2$ implies

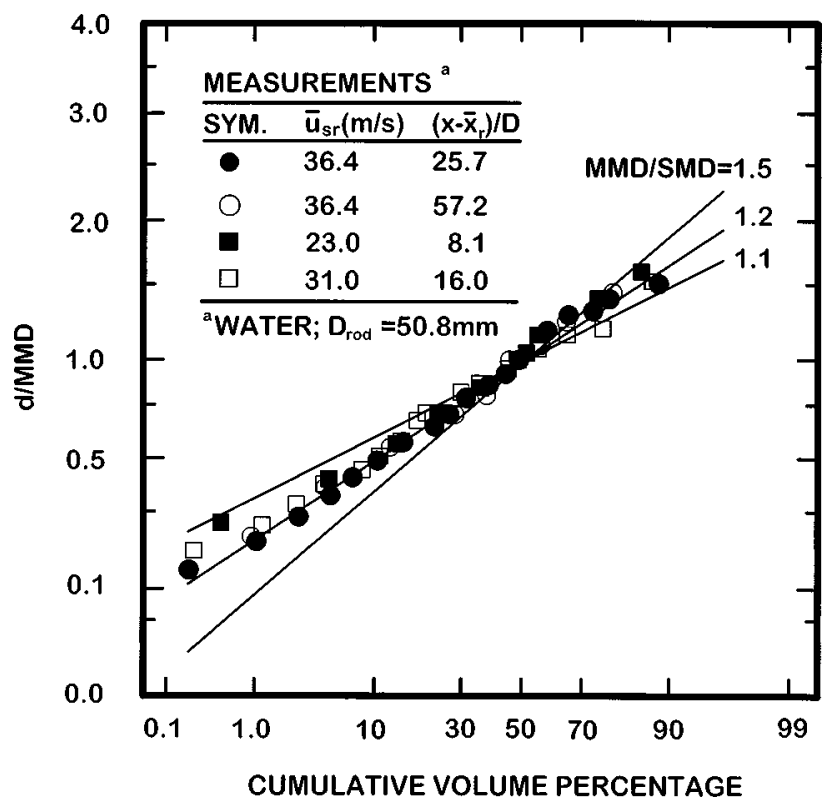

FIG. 7. Distribution of drop diameters after turbulent primary breakup at the free surface of plane wall jets. 


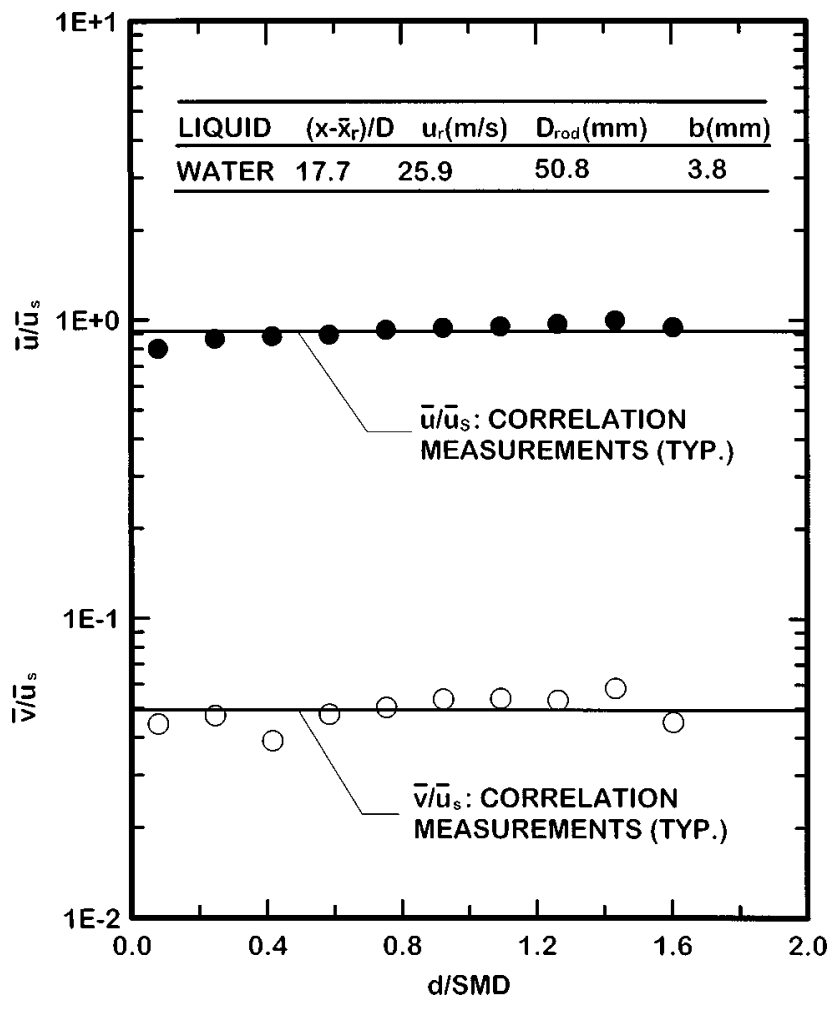

FIG. 8. Distribution of drop velocities after turbulent primary breakup at the free surface of plane wall jets.

that the entire drop size distribution function is known if one other moment, e.g., the SMD, is known. Thus, this property of the root normal distribution function will be exploited in the following with drop sizes characterized by the SMD alone for turbulent primary breakup of plane wall jets similar to results of earlier studies of turbulent primary breakup of round free jets. ${ }^{15}$

\section{E. Drop velocity distributions}

Present measurements of drop velocity distributions after turbulent primary breakup for plane turbulent liquid wall jets are plotted in Fig. 8. The results shown include $\bar{u} / \bar{u}_{s}$ and $\bar{v} / \bar{u}_{s}$ as a function of $d / \mathrm{SMD}$. Ten drop size groups are considered with the velocities of 10-30 drops in each size group averaged to obtain the mean velocities plotted in the figure. It is evident that the results of Fig. 8 are represented reasonably well by universal distribution functions, as follows:

$$
\bar{u} / \bar{u}_{s}=0.92, \quad \bar{v} / \bar{u}_{s}=0.049, \quad 0.08 \leqslant d / \mathrm{SMD} \leqslant 1.6
$$

with standard deviations of the constants on the right-hand sides of Eqs. (6) of $7 \%$ and $12 \%$, respectively. Noting that cross-stream mean velocities in turbulent jets typically are comparable to velocity fluctuations, ${ }^{23}$ the results of Eqs. (6) suggest that the streamwise and cross-stream drop velocities after turbulent primary breakup for plane wall jets are comparable to mean and fluctuating velocities in the liquid phase, which is similar to earlier findings for turbulent primary breakup for round free jets. ${ }^{15-17}$

\section{F. Drop velocities}

Moments of mean and fluctuating drop velocities will be considered next in order to help define the dynamics of turbulent primary breakup before considering the relevant moment of drop sizes, e.g., the SMD. Present measurements of drop velocities after turbulent primary breakup included mean and fluctuating mass-averaged streamwise and crossstream velocities after turbulent primary breakup at various positions along the surface of a plane wall jet. The range of the measurements can be best stated in terms of the streamwise distance variable measured from the onset of a roughened surface, normalized by the radial integral scale, $\Lambda$, and the Weber number based on this scale, $\mathrm{We}_{f \Lambda}=\rho_{f} \bar{u}_{r s}^{2} \Lambda / \sigma$, of the wall jet. This choice of streamwise distance was made for consistency with results for the variation of SMD along the liquid surface to be discussed later, where $\left(x-\bar{x}_{r}\right) /$ $\left(\Lambda \mathrm{We}_{f \Lambda}^{1 / 2}\right)$ will prove to be the "natural" streamwise distance variable. Analogous to earlier work for round turbulent jets ${ }^{15-17}$ the streamwise integral length scale is taken to be equal to $4 \Lambda$ while $\Lambda=D / 8$ based on Laufer's measurements of the properties of fully developed turbulent pipe flow, see Hinze $^{23}$ and references cited therein. It was found that all components and moments of drop velocities after turbulent primary breakup are fixed fractions of local mean streamwise velocities at the liquid surface, independent of position along the surface. This behavior is similar to earlier observations of mean drop velocities after turbulent primary breakup of round turbulent liquid jets due to $\mathrm{Wu}$ and co-workers. ${ }^{15-17}$ The correlations of this behavior for mass-averaged mean velocities are as follows:

$$
\widetilde{u} / \bar{u}_{s}=0.95, \quad \widetilde{v} / \bar{u}_{s}=0.037
$$

with standard deviations of $2 \%$ and $38 \%$, respectively. The corresponding correlations for mass-averaged fluctuating velocities are

$$
\tilde{u}^{\prime \prime} / \bar{u}_{s}=0.056, \quad \widetilde{v}^{\prime \prime} / \bar{u}_{s}=0.020
$$

with standard deviations of $23 \%$ and $20 \%$, respectively. These results were obtained for $\left(x-\bar{x}_{r}\right) /\left(\Lambda \mathrm{We}_{f \Lambda}^{1 / 2}\right)$ in the range $0.15-10$. The values of the moments of mass-averaged mean velocities of Eqs. (7) differ slightly from the results of the individual mean drop velocity distributions of Eqs. (6), while they should be the same because the velocity distribution functions are uniform. This result is reasonable, however, because the differences are well within present experimental uncertainties. Finally, the results of Eqs. (7) and (8) are reasonably consistent with past findings concerning drop velocities after turbulent primary breakup for round free jets where the mean streamwise velocities of the drops were comparable to the mean streamwise velocities at the liquid surface, while mean cross-stream velocities and all components of velocity fluctuations of the drops were comparable to velocity fluctuations at the liquid surface. ${ }^{15-17}$

\section{G. Onset of breakup}

Approximate analysis to find the properties of the onset of turbulent primary breakup was carried out for the plane wall jets using methods similar to earlier considerations of 
the onset of turbulent primary breakup for round free jets. Thus, the process is assumed to involve the formation of a drop from a turbulent eddy near the liquid surface having a characteristic dimension, $l$, and a characteristic cross-stream velocity relative to the surrounding liquid of $v_{l}$. The onset of turbulent primary breakup occurs after the onset of a roughened liquid surface; therefore, the wall jet is reasonably turbulent at the onset of turbulent primary breakup so that the turbulence properties of the wall jet can be taken to be the same as a fully developed turbulent pipe flow having the same hydraulic diameter and $\operatorname{Re}_{f D} \cdot{ }^{19,23}$ Other assumptions were the same as the analysis for the onset of a roughened liquid surface, as follows: Values of the mean streamwise velocity and the thickness of the wall jet were assumed to be constant at $\bar{u}_{s r}$ and $b_{f r}$, respectively; all other physical properties were assumed to be constant; and aerodynamic effects at the liquid surface and during the primary breakup process are assumed to be small. Thus, the eddy was assumed to convect along the surface in the streamwise direction at the local mean velocity, $\bar{u}_{s r}$, while the drop formed by the eddy was assumed to have a diameter comparable to $l$.

The drops formed at the onset of turbulent primary breakup are the smallest drops that can be formed by this mechanism based on both shadowgraph observations and time-scale considerations. The smallest drops that can be formed by turbulent primary breakup, however, are either comparable to the smallest scale of the turbulence (the Kolmogorov microscale) or to the smallest eddy that has sufficient kinetic energy relative to its immediate surroundings to provide the surface energy needed to form a drop, whichever is larger. The Kolgomorov length scale, $l_{K}$, for fully developed turbulent pipe flow can be estimated as follows: ${ }^{24}$

$$
l_{K}=4 \Lambda /\left(4 \Lambda \bar{u}_{r s} / \nu_{f}\right)^{3 / 4} \text {, }
$$

where the streamwise integral length scale has been taken to be equal to $4 \Lambda$, as noted earlier. For present conditions, $l_{K}$ is smaller than $10 \mu \mathrm{m}$ which is much smaller than the smallest drops that were observed at the onset of turbulent primary breakup. Thus, the following analysis will only consider energy requirements to find drop properties at the onset of turbulent primary breakup.

The energy criterion for the smallest drop that can be formed by turbulent primary breakup, which defines conditions at the onset of turbulent primary breakup, is found by equating the kinetic energy of an eddy of characteristic size, $l_{i}$, and velocity, $v_{l i}$, relative to its surroundings, to the surface energy required to form a drop of comparable size, as follows:

$$
\pi \rho_{f} l_{i}^{3} v_{l i}^{2} \sim \pi l_{i}^{2} \sigma
$$

Only a crude proportionality is implied by Eq. (10) due to effects of irregular drop shapes, nonuniform velocities within the eddy and the efficiency of conversion of kinetic energy into surface energy. The largest eddy length scales are somewhat larger than $\Lambda$, while $\Lambda$ corresponds to the largest drop diameter observed during the present investigation. Furthermore, $l_{K}<l_{i}$ for present conditions, as just discussed. Then it

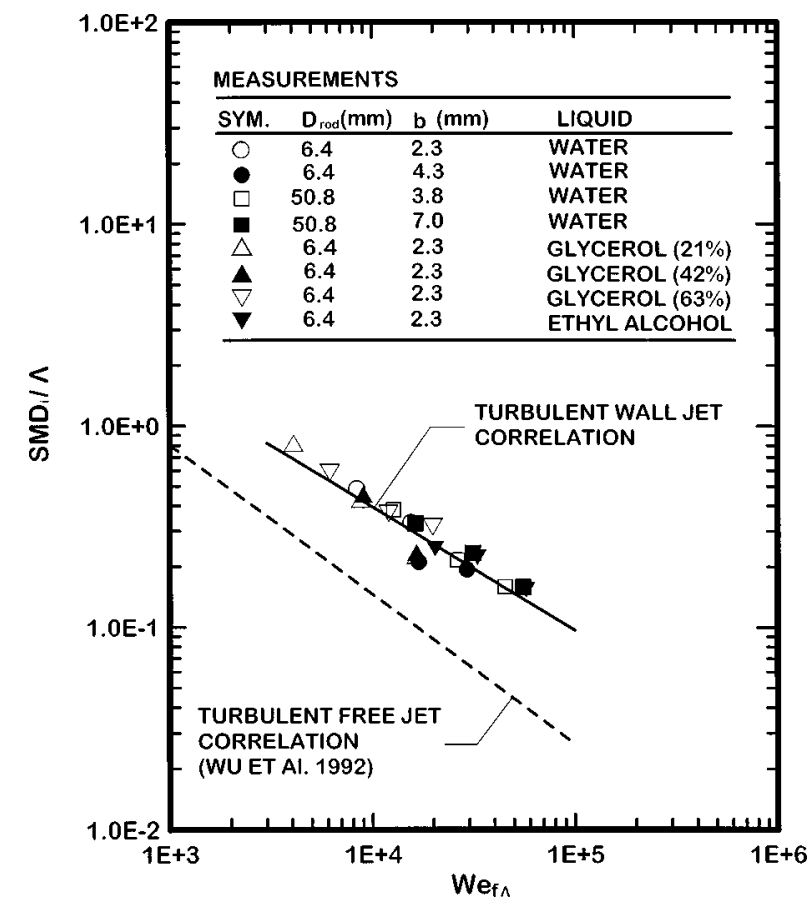

FIG. 9. SMD at the onset of turbulent primary breakup at the free surface of plane wall jets as a function of Weber number.

is reasonable to assume that $l_{i}$ is within the inertial range of the turbulence spectrum, where $l_{i}$ and $v_{l i}$ are related, as follows: ${ }^{24}$

$$
v_{l i} \sim \bar{v}_{s r}^{\prime}\left(l_{i} / \Lambda\right)^{1 / 3}
$$

Variations of turbulence properties within the liquid are ignored when using Eq. (11), similar to earlier considerations of turbulent primary breakup for round free jets. ${ }^{15-17}$ Substituting Eq. (11) into Eq. (10), setting $\mathrm{SMD}_{i} \sim l_{i}$ and assuming that wall jet turbulence properties can be approximated by the properties of fully developed turbulent pipe flow having a mean streamwise velocity, diameter and Reynolds number of $\bar{u}_{s r}, D$ and $\mathrm{Re}_{f D}$, the expression for $\mathrm{SMD}_{i}$ becomes

$$
\mathrm{SMD}_{i} / \Lambda=C_{s i}\left(\bar{u}_{s r} / \bar{v}_{s r}^{\prime}\right)^{6 / 5} \mathrm{We}_{f \Lambda}^{-3 / 5},
$$

where $C_{s i}$ is an empirical constant of order unity that involves the various constants of proportionality of the analysis. For fully developed turbulent pipe flow, $\bar{v}_{s r}^{\prime} \bar{u}_{s r}$ is a constant; therefore, $\mathrm{SMD}_{i} / \Lambda$ should only be a function of $\mathrm{We}_{f \Lambda}$ for the present test conditions.

The present measurements of $\mathrm{SMD}_{i}$ for plane wall jets are plotted in terms of the variables of Eq. (12) in Fig. 9, along with an earlier correlation measured by $\mathrm{Wu}$ and Faeth $^{15}$ for round free jets. The correlation of the present measurements for plane wall jets in these coordinates is well within the scatter anticipated based on experimental uncertainties. It is also helpful that the present broad range of aspect ratios correlate in the same manner in terms of $\Lambda$ (and thus the hydraulic diameter $D$ ). The present correlation of $\mathrm{SMD}_{i}$ for the wall jets yields the following empirical fit that is shown on the plot:

$$
\mathrm{SMD}_{i} / \Lambda=109 \mathrm{We}_{f \Lambda}^{-0.61} \text {. }
$$


The standard deviations of the coefficient and power of Eq. (13) are $15 \%$ and $8 \%$, respectively, while the correlation coefficient of the fit is 0.89 . The differences among the power of the best fit of the data, -0.61 , the power predicted by the simplified theoretical result in Eq. (12), -0.60 and the earlier correlation for round free jets, ${ }^{15}-0.74$ are not statistically significant. The coefficient of Eq. (13) is relatively large but this is expected from Eq. (12) because $\left(\bar{u}_{s r} / \bar{v}_{s r}^{\prime}\right)^{6 / 5}$ is relatively large; therefore, $C_{s i}$ is of order unity as anticipated for an empirical parameter of this type. Finally, the present correlation for wall jets gives values of $\mathrm{SMD}_{i}$ that are of the same order of magnitude but larger than the earlier results for round free jets at comparable conditions. Nevertheless, differences of this magnitude might be anticipated due to the different turbulence states of these flows and when concepts of hydraulic diameters are used to estimate integral scales of turbulence and to compare findings for plane wall jets and round free jets.

The position of the onset of turbulent primary breakup for plane wall jets was also found similar to the earlier study of round free jets. It was assumed that the eddy that forms the drops at the onset of turbulent primary breakup convects along the liquid surface with a streamwise velocity $\bar{u}_{s r}$ for a time $\tau_{i}$ required for an eddy having characteristic size $l_{i}$ to form a drop. There are several characteristic breakup times that could be used to estimate $\tau_{i}$ as discussed by $\mathrm{Wu}$ and Faeth; ${ }^{15,16}$ based on these considerations, the Rayleigh breakup time was chosen for the present analysis. Thus, ignoring effects of liquid viscosity on the Rayleigh breakup time, which would be required at larger Ohnesorge number conditions as discussed by Weber, ${ }^{25}$ the expression for $\tau_{i}$ becomes $^{15}$

$$
\tau_{i} \sim\left(\rho_{f} l_{i}^{3} / \sigma\right)^{1 / 2} .
$$

This breakup time comes about as a result of interactions between inertial and surface tension forces so that it is independent of $v_{l i}$. The distance required for the onset of turbulent primary breakup is measured relative to the onset of surface roughness, as follows:

$$
\bar{x}_{i}-\bar{x}_{r} \sim \bar{u}_{s r} \tau_{i} .
$$

An expression for $\bar{x}_{i}-\bar{x}_{r}$ is then found by substituting Eq. (14) into Eq. (15) and letting $\mathrm{SMD}_{i} \sim l_{i}$, as follows:

$$
\left(\bar{x}_{i}-\bar{x}_{r}\right) / \Lambda \sim\left(\mathrm{SMD}_{i} / \Lambda\right)^{3 / 2} \mathrm{We}_{f \Lambda}^{1 / 2} .
$$

Finally, eliminating $\mathrm{SMD}_{i}$ from Eq. (16), using Eq. (12), yields an expression for the location of the onset of turbulent primary breakup, as follows:

$$
\left(\bar{x}_{i}-\bar{x}_{r}\right) / \Lambda=C_{x i}\left(\bar{u}_{s r} / \bar{v}_{s r}^{\prime}\right)^{9 / 5} \mathrm{We}_{f \Lambda}^{-0.4},
$$

where $C_{x i}$ is the constant of proportionality of order of magnitude unity while $\bar{v}_{s r}^{\prime} / \bar{u}_{s r}$ is a constant for fully developed plane turbulent wall jets.

Present measurements of $\bar{x}_{i}-\bar{x}_{r}$ are plotted in terms of the variables of Eq. (17) in Fig. 10, along with the earlier correlation of $\bar{x}_{i} / \Lambda$ found by Wu et al. ${ }^{15}$ for round free jets (note that $\bar{x}_{r} \approx 0$ for round free jets). The correlation of the present measurements in these coordinates is similar to the earlier results for free round jets and is within the scatter

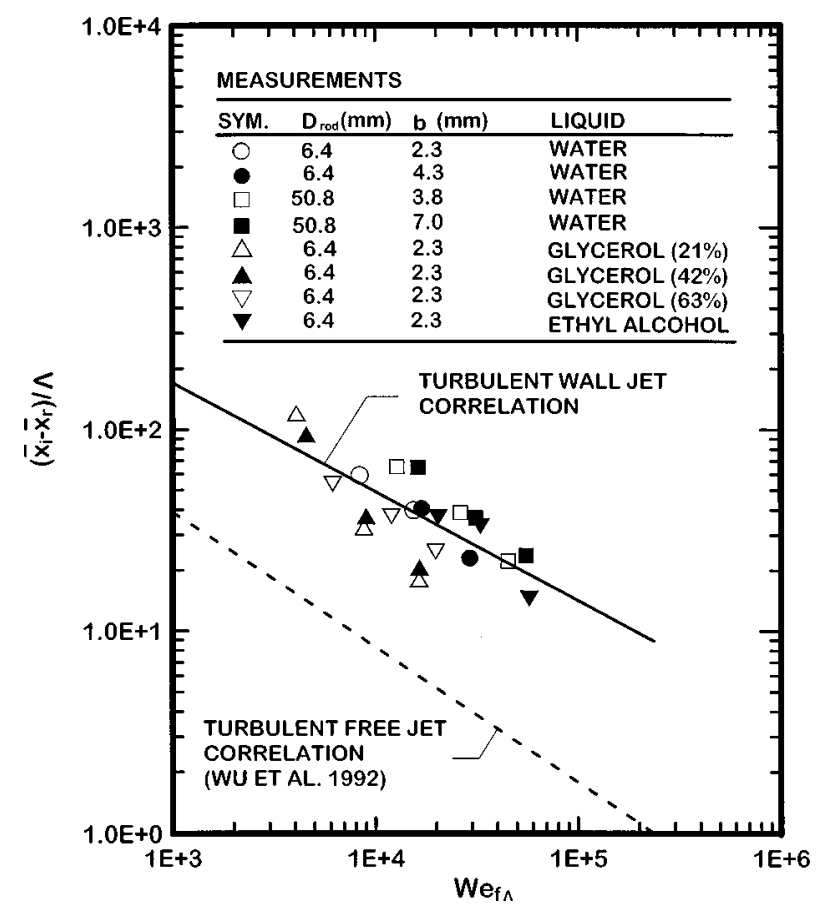

FIG. 10. Length to initiate turbulent primary breakup at the free surface of plane wall jets as a function of Weber number.

expected based on experimental uncertainties. The power of $\mathrm{We}_{f \Lambda}$ for the present data correlation is not -0.4 as suggested by Eq. (17), however, and can be represented better by the following empirical fit that is shown on the plot:

$$
\left(\bar{x}_{i}-\bar{x}_{r}\right) / \Lambda=7100 \mathrm{We}_{f \Lambda}^{-0.54} \text {. }
$$

The standard deviations of the coefficient and power of Eq. (18) are $35 \%$ and $19 \%$, respectively, while the correlation coefficient of the fit is 0.57 . The present power of $\mathrm{We}_{f \Lambda}$, -0.54 , falls between the power predicted by the theoretical result in Eq. (17), - 0.4, and the earlier correlation for round free jets, ${ }^{15}-0.67$, but these differences are not statistically significant due to the scatter of the data. As before, the large value of the coefficient on the RHS of Eq. (18) can be anticipated from Eq. (17) because $\left(\bar{u}_{s r} / \bar{v}_{s r}^{\prime}\right)^{9 / 5}$ is large for typical turbulent pipe flows

\section{H. Drop sizes}

The approach used to find an expression for the variation of SMD with distance from the onset of roughness was similar to the method used to find $\bar{x}_{i}-\bar{x}_{r}$. First of all, it is reasonable to assume that drops near the liquid surface have been formed recently by primary breakup because they have relatively large cross-stream velocities independent of size, see Eqs. (6) and (7). It is also reasonable to assume that the SMD is dominated by the largest drops in the drop size distribution so that the SMD is proportional to the largest drop that can be formed at a particular position. Then, neglecting variations of wall jet velocities, as before, $\left(x-\bar{x}_{r}\right) \sim \bar{u}_{s r} \tau$ where the breakup time, $\tau$, is a function of the SMD. Finally, adopting the Rayleigh breakup mechanism and letting $\mathrm{SMD} \sim l$, as before, a procedure similar to the derivation of 


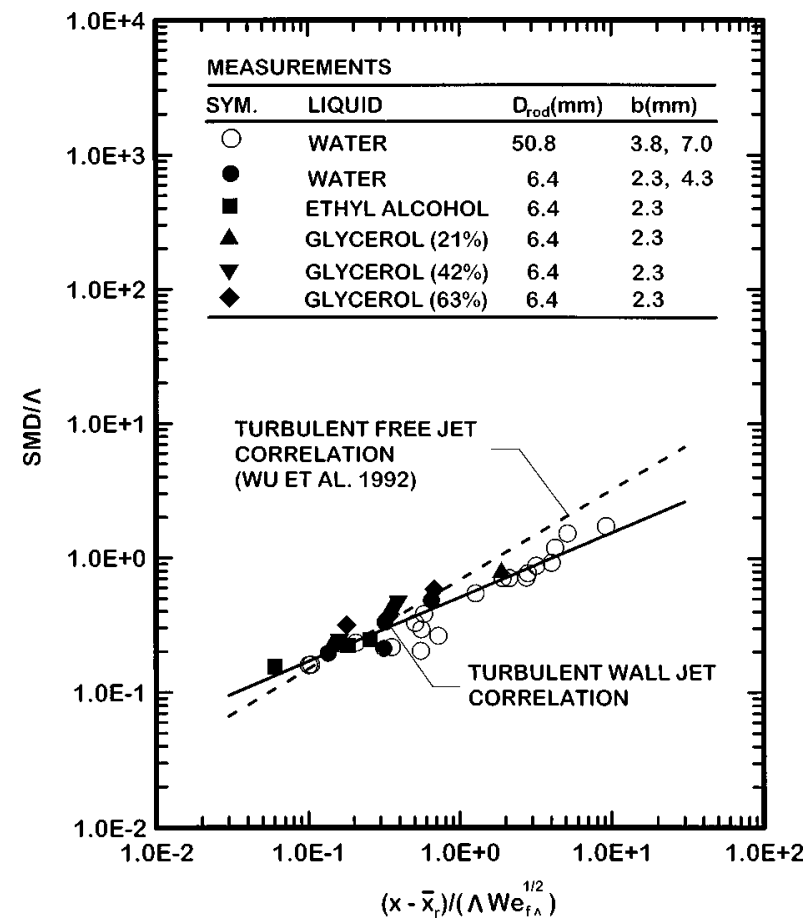

FIG. 11. SMD after turbulent primary breakup at the free surface of plane wall jets as a function of distance from the onset of surface roughness.

Eq. (16) yields the following expression for the variation of SMD with distance from the onset of surface roughness:

$$
\mathrm{SMD} / \Lambda=C_{s x}\left(\left(x-\bar{x}_{r}\right) /\left(\Lambda \mathrm{We}_{f \Lambda}^{1 / 2}\right)\right)^{2 / 3},
$$

where $C_{s x}$ is a constant of proportionality that should be on the order of unity. The approach used to derive Eq. (16) was essentially the same as the approach used to relate $\left(\bar{x}_{i}-\bar{x}_{r}\right)$ and $\mathrm{SMD}_{i}$ and should yield the same result; comparing Eqs. (16) and (19) demonstrates that this is properly the case.

The present measurements of SMD for plane wall jets are plotted in terms of the variables of Eq. (19) in Fig. 11, along with the earlier correlation measured by Wu et al. ${ }^{15}$ for round free jets. The correlation of the present measurements for plane wall jets in these coordinates is well within the scatter anticipated based on experimental uncertainties. As before, it is helpful that aspect ratios of $D_{\text {rod }} / b_{f r}$ in the range 1.5-13.4 correlate in an identical manner in terms of a radial integral scale, $\Lambda$, found from hydraulic diameter concepts. The present best fit correlation for the variation of SMD with distance for plane wall jets becomes

$$
\mathrm{SMD} / \Lambda=0.51\left(\left(x-\bar{x}_{r}\right) /\left(\Lambda \mathrm{We}_{f \Lambda}^{1 / 2}\right)\right)^{0.48} .
$$

The standard deviations of the coefficient and power of Eq. (20) are $24 \%$ and $6 \%$, respectively, and the correlation coefficient of the fit is 0.88 . The differences among the power of the best fit of the data, 0.48 , the power predicted by the simplified theoretical result in Eq. (19), 0.67, and the earlier correlation for round free jets, ${ }^{15} 0.67$, are statistically significant. These differences are not large, however, in view of the different turbulent states of decaying free round turbulent free jets and developing plane turbulent wall jets. Finally, the coefficient of Eq. (20), $C_{s x}$, is on the order of unity as expected because there is no term proportional to $\bar{u}_{s r} / \bar{v}_{s r}^{\prime}$ which becomes large in this case. Taken together, the fact that $C_{s i}, C_{x i}$ and $C_{s a}$ all had reasonable magnitudes, on the order of unity, while large values of the coefficients on the RHS of the correlation expressions were properly associated with the ratio of $\bar{u}_{s r} / \bar{v}_{s r}^{\prime}$ to some power, which generates large numbers, helps support the physical ideas that were the basis of the various expressions used to interpret and correlate the present measurements.

It should be noted that increasing distance will eventually yield a condition where the SMD approaches $\Lambda$ according to either Eq. (20) or the result plotted in Fig. 11. This condition causes two types of behavior for round free jets: (1) breakup of the entire liquid column at conditions similar to those observed by Grant and Middleman, ${ }^{8}$ for round turbulent free jets in still gases, and (2) transition to the largeeddy subrange of the three-dimensional turbulent energy spectrum leading to termination of turbulent primary breakup along the liquid surface, similar to behavior observed by $\mathrm{Wu}$ and Faeth. ${ }^{17}$ The corresponding behavior for plane wall jets is not known and clearly merits attention in the future.

\section{CONCLUSIONS}

The properties of the liquid surface, and of drop sizes after turbulent primary breakup along the liquid surface, were measured for plane liquid wall jets along smooth walls in still air at normal temperature and pressure. Experimental conditions included water, various glycerol mixtures and ethyl alcohol wall jets having $\rho_{f} / \rho_{g}=680-980, \operatorname{Re}_{f D}$ $=17000-840000, \quad \mathrm{We}_{f D}=6100-57000$ and $\mathrm{Oh}_{f D}$ $=0.001-0.013$, the last implying conditions where direct effects of liquid viscosity are small. The major conclusions of the study are as follows:

(1) The presence of roughness at the liquid surface, and turbulent primary breakup along the liquid surface, were caused by turbulence due to liquid motion past the wall, while direct effects of aerodynamic forces at the liquid surface were small, for present conditions.

(2) Transition to a roughened liquid surface occurred when the outer edge of the turbulent boundary layer developing along the wall approached the liquid surface. For present conditions, where turbulent boundary layer growth was initiated by a trip wire, distances to the onset of a roughened liquid surface could be correlated by the turbulent boundary layer thickness expression of Schlichting. ${ }^{19}$

(3) Drop sizes after turbulent primary breakup satisfied the universal root normal distribution function with $\mathrm{MMD} / \mathrm{SMD}=1.2$ due to Simmons, ${ }^{20}$ similar to past observations of a variety of primary and secondary breakup processes, see Refs. 13-17; this behavior is helpful because the complete drop size distribution is then fully specified by a single parameter, e.g., by the SMD. Corresponding drop velocities after turbulent primary breakup were essentially independent of drop size, i.e., they satisfied the uniform distribution function.

(4) The mass-averaged streamwise velocities of drops after turbulent primary breakup were comparable to mean streamwise velocities in the wall jet while mass- 
averaged velocity fluctuations and mean cross-stream velocities of drops after turbulent primary breakup were comparable to velocity fluctuations in the wall jet.

(5) Drop sizes at the onset of turbulent primary breakup along the liquid surface could be correlated by equating the surface energy required to form a drop to the kinetic energy of an eddy of corresponding size within the inertial range of the turbulence spectrum. Onset of turbulent primary breakup could also be controlled by the smallest (Kolmogorov) and largest turbulence scales in the liquid but such conditions were not observed during the present investigation.

(6) The position of the onset of turbulent primary breakup along the liquid surface could be correlated by considering the distance convected at the mean velocity of the wall jet for the residence time needed to initiate Rayleigh breakup of the ligaments protruding from the liquid surface that are associated with the formation of drops of similar size at the onset of breakup.

(7) Drop sizes after turbulent primary breakup progressively increased with increasing distance along the liquid surface. The variation of drop sizes with distance was virtually the same as at the onset of turbulent primary breakup and could be explained by associating the SMD with the largest drops that had sufficient residence time in the flow to be formed at a particular location due to Rayleigh breakup of protruding ligaments of similar size.

(8) The general behavior of turbulent primary breakup observed during the present study of turbulent wall jets was similar to earlier observations of turbulent primary breakup in round free jets, see Refs. 15-17, except for obvious differences due to different turbulence properties. In both cases, consideration of conditions where effects of liquid viscosities are more important, where the onset of drop breakup approaches the smallest (Kolmogorov) and largest scales of the liquid phase turbulence, where drop sizes due to breakup approach the wall jet height and where aerodynamic effects become important, clearly merit more attention.

\section{ACKNOWLEDGMENTS}

This research was supported by the Office of Naval Research, Grant No. N00014-95-1-0234 under the technical management of E. P. Rood. The help of L.-P. Hsiang during the early phases of the study is gratefully acknowledged.
${ }^{1}$ M. Gad-el-Hak, "Measurements of turbulence and wave statistics in windwaves," International Symposium on Hydrodynamics in Ocean Engineering (The Norwegian Institute of Technology, Olso, Norway, 1981), pp. 403-417.

${ }^{2}$ J. M. Townson, Free-Surface Hydraulics, 1st ed. (Unwin Hyman, London, 1988), Chap. 6.

${ }^{3}$ D. A. Ervine and H. T. Falvey, "Behavior of turbulent water jets in the atmosphere and in plunge pools," Proc. Inst. Civ. Eng. 83 (Pt. 2), 295 (1987).

${ }^{4}$ P.-K. Wu, R. F. Miranda, and G. M. Faeth, "Effects of initial flow conditions on primary breakup of nonturbulent and turbulent round liquid jets,' Atomization Sprays 5, 175 (1995).

${ }^{5}$ K. J. De Juhasz, O. F. Zahn, Jr., and P. H. Schweitzer, “'On the formation and dispersion of oil sprays,', Bulletin No. 40, Engineering Experimental Station, Pennsylvania State University, p. 63, 1932.

${ }^{6}$ D. W. Lee and R. C. Spencer, "Preliminary photomicrographic studies of fuel sprays,', NACA Tech. Note 424, Washington, D.C. 1933.

${ }^{7}$ D. W. Lee, and R. C. Spencer, "Photomicrographic studies of fuel sprays," NACA Tech. Note 454, Washington, D.C. 1933.

${ }^{8}$ R. P. Grant and S. Middleman, "Newtonian jet stability,', AIChE J. 12, 669 (1966)

${ }^{9}$ R. E. Phinney "The breakup of a turbulent jet in a gaseous atmosphere," J. Fluid Mech. 60, 689 (1973).

${ }^{10}$ M. J. McCarthy and N. A. Malloy, "Review of stability of liquid jets and the influence of orifice design,', Chem. Eng. J. 7, 1 (1974).

${ }^{11}$ J. W. Hoyt and J. J. Taylor, "Waves on water jets,', J. Fluid Mech. 88, 119 (1977).

${ }^{12}$ J. W. Hoyt and J. J. Taylor, "Turbulence structure in a water jet discharging in air,' Phys. Fluids 20, S253 (1977).

${ }^{13}$ G. A. Ruff, P.-K. Wu, L. P. Bernal, and G. M. Faeth, "Continuous- and dispersed-phase structure of dense non-evaporating pressure-atomized sprays,', J. Propul. Power 8, 280 (1992).

${ }^{14}$ L.-K. Tseng, G. A. Ruff, and G. M. Faeth, 'Effects of gas density on the structure of liquid jets in still gases," AIAA J. 30, 1537 (1992).

${ }^{15}$ P.-K. Wu, L.-K. Tseng, and G. M. Faeth, "Primary breakup in gas/liquid mixing layers for turbulent liquids,' Atomization Sprays 2, 295 (1992).

${ }^{16}$ P.-K. Wu and G. M. Faeth, "Aerodynamic effects on primary breakup of turbulent liquids,' Atomization Sprays 3, 265 (1993).

${ }^{17}$ P.-K. Wu and G. M. Faeth, "Onset and end of drop formation along the surface of turbulent liquid jets in still gases,' Phys. Fluids 7, 2915 (1995).

${ }^{18}$ Z. Dai, L.-P. Hsiang and G. M. Faeth, "Spray formation at the free surface of turbulent bow sheets," Twenty-First Symposium on Naval Hydrodynamics (National Academy Press, Washington, D.C., 1997), pp. 490-505.

${ }^{19}$ H. Schlichting, Boundary Layer Theory, 7th ed. (McGraw-Hill, New York, 1979), p. 599.

${ }^{20}$ H. C. Simmons, "The correlation of drop-size distributions in fuel orifice sprays," J. Eng. Power 99, 309 (1977).

${ }^{21}$ L.-P. Hsiang and G. M. Faeth, "Near-limit drop deformation and secondary breakup,' International Journal of Multiphase Flow 18, 635 (1992).

${ }^{22}$ L.-P. Hsiang and G. M. Faeth, "Drop properties after secondary breakup,' International Journal of Multiphase Flow 19, 721 (1993).

${ }^{23}$ J. O. Hinze, Turbulence, 2nd ed. (McGraw-Hill, New York, 1975), pp. 427 and 724-742.

${ }^{24}$ H. Tennekes and J. L. Lumley, A First Course in Turbulence, 1st ed. (MIT Press, Cambridge, MA, 1972), pp. 248-286.

${ }^{25}$ C. Weber, "Zum zerfall eines flussigkeitsstrahles,' Z. Angew. Math. Mech. 2, 136 (1931). 